\title{
Constrained Power Focusing in Inhomogeneous Media as a Polarization Optimization
}

\author{
Domenica Anna Maria Iero \\ Dipartimento dell'Informazione, delle Infrastrutture e dell'Energia Sostenibile, (DIIES), \\ Università "Mediterranea" di Reggio Calabria, Via Graziella, Località Feo di Vito, 89124 Reggio di Calabria, Italy \\ Correspondence should be addressed to Domenica Anna Maria Iero; domenica.iero@unirc.it
}

Received 5 August 2015; Accepted 5 October 2015

Academic Editor: Atsushi Mase

Copyright (C) 2015 Domenica Anna Maria Iero. This is an open access article distributed under the Creative Commons Attribution License, which permits unrestricted use, distribution, and reproduction in any medium, provided the original work is properly cited.

\begin{abstract}
The problem of focusing a field into inhomogeneous media is a canonic open problem relevant in many engineering areas. Several approaches have been developed, for example, Time Reversal, Inverse Filter, and Eigenvalues approach, but they suffer from several drawbacks which are counteracted by Optimal Constrained Power Focusing (OCPF) technique. OCPF was first introduced to deal with scalar fields and, recently, it has been extended to tackle the problem of focusing vector fields. In particular, the proposed approach allows reducing the focusing problem, which is NP-hard in case of vector fields, to a series of convex programming (CP) ones. In this paper, an alternative OCPF formulation is presented, which consists in the research of the most suitable polarization of the field into the target point and relies on the convexity of the problem when fixing this polarization. Such a result allows the development of two different solution procedures: an enumerative one that can take advantage of parallel programming in order to explore all possible polarizations and hybrid one which relies on the exploitation of global search algorithm just to solve the nonconvex part of the problem at hand.
\end{abstract}

\section{Introduction}

Focusing a wave in an inhomogeneous medium is a canonic and general problem which is relevant in many applications, including microwave hyperthermia [1], lithotripsy [2], targeted drug delivery [3], and imaging [4]. Remarkable efforts have been devoted to the development of radiating devices able to produce a field well focused in a target point, stressing the advantages of using array applicators [1] in terms of focusing performance and apparatus' customizability. As a consequence, several focusing procedures based on the suitable array antennas design have been developed, such as Time Reversal [5], Inverse Filter [6], and Eigenvalues approach [7].

These approaches are very efficient but they suffer from the inability of performing a customizable spatial power/field shaping by imposing suitable upper bound constraints, as it is instead achieved by the Optimal Constrained Power Focusing (OCPF) approach $[8,9]$. In particular, Time Reversal completely lacks this capability and, relying on the time invariance of the wave equation which holds true just in lossless media, its focusing performances worsen in lossy media considerably. Obviously some compensation techniques $[4,10]$ have been developed to counteract the effect of losses in the wave propagation but they do not provide any capability of controlling the power/field intensity in the scenario of interest. Such a capability is instead crucial in some applications like hyperthermia [1], and, more in general, it affects the focusing performances in terms of side lobe level remarkably.

Inverse Filter $[6,11]$ takes partially into account this aspect of the focusing problem as it relies on the enforcement of a specific field pattern, which ideally should be a Dirac function of the space coordinates centered at the target point. In this respect, the selection of the reference pattern is crucial and affects the focusing performances so that obtained focused field (which is unavoidably different from the target one) will be reference pattern dependent. Also note that enforcing a perfect match between the actual and reference pattern could be overrestrictive and the actually optimal field to be pursued is not a priori known. 
Differently from the above approaches, the Eigenvalue based focusing technique [7] relies on the formulation of the focusing problem as the maximization of the ratio of two quadratic forms, for example, the energy transmitted into the focus area and the overall transmitted energy.

As in the previously mentioned approaches, the basic version of this focusing strategy [7] does not perform a customizable power deposition spatial shaping, but this capability has been introduced in its adaptive version [12] which iteratively tries to keep under control the highest side lobes by minimizing them in the following iteration. However, by doing so hot spots may simply move from some points to other ones, potentially leading to an endless iterative procedure.

It is also worth noting that none of the mentioned focusing techniques guarantees the optimality of the solution found, which means that, in case of fixed array geometry, there could be a set of array's excitations which provides better focusing capabilities.

In case of scalar fields, OCPF relies on the formulation of the focusing problem as a convex programming $(\mathrm{CP})$ one, which allows the enforcement of upper bound constraints on the power deposition everywhere but in the focal area, guaranteeing the optimality of the solution. On the other side, such a simple result cannot be generalized in a simple fashion to the case of vector fields. In fact, when dealing with vector fields, for example, electromagnetic fields, the cost function to be maximized (or minimized) is not any more a linear function of the unknowns, so that the problem is NP-hard [13].

In this paper, a novel insight into the problem is given. It provides a very simple generalization of the basic approach applied to scalar fields. In particular, it is shown that the noconvex part of the synthesis problem consists in the selection of the field polarization in the target point which provides the highest intensity of the overall field herein. In fact, once the optimal polarization is known, the focusing problem becomes a simple CP one.

This interesting result allows the development of two possible solution strategies. In fact, the overall problem can be recast as the solution of several $\mathrm{CP}$ problems corresponding to any possible field polarization into the target point and by selecting the solution which guarantees the best focusing performances. This focusing procedure can be performed in an enumerative fashion by exploiting parallel programming.

The alternative is searching the optimal polarization by means of global optimization procedures [14]. In this case, global optimization algorithms would be applied to a very small number of parameters (i.e., those identifying the polarization in the target point) so that the issues related to the number of variables, such as the actual optimality of the solution found and the computational burden, are avoided.

The paper is organized as follows. In Section 2, a brief review of OCPF applied to scalar fields is given because it is preparatory for the development of an innovative easyto-understand and easy-to-use OCPF formulation for vector fields, presented in Section 3. For the sake of simplicity, we refer throughout to $z$-invariant scatterers and to focalization of the electric field intensity, so that the scalar problem corresponds to the $\mathrm{TM}_{z}$ polarization and the vector one to the case of $\mathrm{TE}_{z}$ polarization. A numerical assessment is finally given in Section 4 with reference to biomedical applications and hyperthermic cancer treatment [1]. Conclusions follow.

\section{Optimal Constrained Power Focusing of Scalar Fields}

Let us consider an array, made by $N$ antennas, surrounding a domain of interest $\Omega$, wherein the target point is located. The field one wants to shape is given by

$$
E(\underline{r})=\sum_{n=1}^{N} I_{n} \varphi_{n}(\underline{r}),
$$

where $\varphi_{n}$ represents the scalar total field arising in $\Omega$, in TM polarization, when only the $n$th antenna is fed (by unitary excitation), $I_{n}$ is the actual complex excitation of the $n$th antenna, and, for the sake of simplicity, monochromatic excitations are considered for the time being.

The focusing problem can be formulated as the maximization of $|E(\underline{r})|^{2}$ in the target point $\underline{r}_{T}$, while bounding the power deposition elsewhere. Fixing the applicator geometry, this focusing problem is similar to the classical synthesis of pencil beams by means of array antennas $[15,16]$ and, hence, can be recast as follows.

Determine the real and imaginary parts of the array excitation coefficients $\mathfrak{R}\left\{I_{n}\right\}$ and $\mathfrak{\Im}\left\{I_{n}\right\}(n=1 \cdots N)$ such as

$$
\begin{aligned}
\max & \Re\left\{E\left(\underline{r}_{T}\right)\right\} \\
\text { subject to: } & \mathfrak{J}\left\{E\left(\underline{r}_{T}\right)\right\}=0 \\
& |E(\underline{r})|^{2} \leq \mathscr{U} \mathscr{B}(\underline{r}) \quad \underline{r} \in \Omega \backslash \Pi\left(\underline{r}_{T}\right) .
\end{aligned}
$$

In $(2 \mathrm{c}), \mathcal{U} \mathscr{B}(\underline{r})$ is a (nonnegative and arbitrary) "mask" function that enforces upper bound constraints on the power distribution. It is set according to the specific application's requirements. The constraints are meant to keep the maximum power level bounded everywhere in $\Omega$ but for a neighborhood of the target point, that is, the focal area $\Pi\left(\underline{r}_{T}\right)$.

Notably, thanks to a proper choice of the reference phase, the cost function has turned into a linear function of the real and imaginary parts of the excitations. As constraints (2b) and $(2 c)$ are convex with respect to the unknowns $I_{n}$, the focusing problem is now formulated in terms of a convex programming $(\mathrm{CP})$ one $[8,9]$, whose solution is globally optimal and it can be found by local search algorithm with little computational effort. Moreover, even if herein it has been developed with respect to a $\mathrm{TM}_{z}$ polarized electric field in a $z$-invariant scenario, the above formulation is general and can be applied to any kind of field as long as it is scalar.

\section{Optimal Constrained Power Focusing of Vector Fields}

Apart from special cases, in electromagnetics the field one wants to shape has a vector nature, which introduces a further difficulty to the focusing problem as the optimization of the 
field intensity cannot be reduced anymore (as we have done in Section 2) to the optimization of a simple linear function.

In this case, considering an array made by $N$ antennas surrounding a domain of interest $\Omega$, the field, whose intensity has to be shaped, is given by

$$
\mathbf{E}(\underline{r})=\sum_{n=1}^{N} I_{n} \Phi_{n}(\underline{r}),
$$

where $I_{n}$ is the actual complex excitation of the $n$th antenna and $\Phi_{n}$ represents the vector total field arising in $\Omega$ when only the $n$th antenna is fed (by unitary excitation).

For the sake of simplicity, monochromatic excitations and $\mathrm{TE}_{z}$ polarized fields in a $z$-invariant scenario $\Omega$ are considered, so that the intensity of the total field computed into the target point $\underline{r}_{T}$ can be expressed as [17]

$$
\begin{aligned}
\mathbf{E}\left(\underline{r}_{T}\right) & =\sum_{n=1}^{N} I_{n}\left\langle\Phi_{n}\left(\underline{r}_{T}\right), \mathbf{p}\right\rangle \mathbf{p}+\sum_{n=1}^{N} I_{n}\left\langle\Phi_{n}\left(\underline{r}_{T}\right), \mathbf{q}\right\rangle \mathbf{q} \\
& =\sum_{n=1}^{N} I_{n} \varphi_{p n}\left(\underline{r}_{T}\right) \mathbf{p}+\sum_{n=1}^{N} I_{n} \varphi_{q n}\left(\underline{r}_{T}\right) \mathbf{q},
\end{aligned}
$$

where $\mathbf{p}$ and $\mathbf{q}$ are two generic orthogonal polarizations of the field (e.g., the two circular polarizations or two orthogonal linear ones or whatever).

As in the previous case of scalar fields, the focusing problem can be formulated as the maximization of $|\mathbf{E}(\underline{r})|^{2}$ in the target point $\underline{r}_{T}$, while bounding the power deposition elsewhere. This optimization problem is nonlinear [13], as the objective function $\left|\mathbf{E}\left(r_{T}\right)\right|^{2}$ is a nonnegative quadratic polynomial with respect to the unknown coefficients $\mathbf{I}_{n}$. Hence, the overall optimization problem belongs to the class of NP-hard problems [18] and a specific strategy is required to cope with it.

If the polarization (let us say $\widehat{\mathbf{p}}$ ) which guarantees the highest $\left|\mathbf{E}\left(\underline{r}_{T}\right)\right|^{2}$ value was a priori known, the corresponding field into the target point would be

$$
\mathbf{E}\left(\underline{r}_{T}\right)=\sum_{n=1}^{N} I_{n}\left\langle\Phi_{n}\left(\underline{r}_{T}\right), \widehat{\mathbf{p}}\right\rangle \widehat{\mathbf{p}}=\sum_{n=1}^{N} I_{n} \varphi_{\widehat{p} n}\left(\underline{r}_{T}\right) \widehat{\mathbf{p}}
$$

as only the first term at the right hand side of (4) has to survive if the field into the target point is optimally $\widehat{\mathbf{p}}$ polarized. Under such a circumstance, by using the same kind of reasoning as in Section 2, the overall problem could be finally turned into

$$
\max \Re\left\{\sum_{n=1}^{N} I_{n} \varphi_{\widehat{p} n}\left(\underline{r}_{T}\right)\right\}
$$

subject to: $\quad \mathfrak{S}\left\{\sum_{n=1}^{N} I_{n} \varphi_{\widehat{p} n}\left(\underline{r}_{T}\right)\right\}=0$

$$
\left|\sum_{n=1}^{N} I_{n} \Phi_{n}(\underline{r})\right|^{2} \leq \mathcal{U} \mathscr{B}(\underline{r})
$$

As constraint (6c) is a quadratic semidefinite function of $I_{n}$ and since (6b) and the cost function (6a) are linear with respect to the unknowns, the knowledge of $\widehat{\mathbf{p}}$ would allow reducing the overall focusing problem to a $\mathrm{CP}$ one.

Unfortunately, it is impossible to a priori know the optimal polarization $\widehat{\mathbf{p}}$. On the other side, the polarization of a field is determined by few parameters, so that the very simple idea, suggested here, is to introduce an optimization procedure looking for the best polarization $\widehat{\mathbf{p}}$.

Then the so-defined optimization problem can be solved by changing in an enumerative fashion the target polarization and solving the corresponding CP problem. By doing so, one gets several sets of optimal candidate excitations among which one can select those providing the maximum $|\mathbf{E}(r)|^{2}$ into the target point $\underline{r}_{T}$, that is, the globally best solution. Such a formulation allows exploiting parallel programming toolboxes to speed up the overall focusing procedure as the solution of each CP problem corresponding to a fixed field polarization $\mathbf{p}$ is independent from the others and when considering a huge number of candidate polarizations the overall optimization procedure could be time consuming even if the solution of each CP problem is very efficient.

An alternative strategy, inspired by [19] and aimed at avoiding the previous enumerative procedure, consists in performing an optimization in the space of polarizations. In this respect, let us consider the problem

$$
\begin{aligned}
& \max \quad \mathfrak{R}\left\{\sum_{n=1}^{N} I_{n} \varphi_{p n}\left(\underline{r}_{T}\right)\right\} \\
& \text { subject to } \quad \mathfrak{\Im}\left\{\sum_{n=1}^{N} I_{n} \varphi_{p n}\left(\underline{r}_{T}\right)\right\}=0 \\
& \sum_{n=1}^{N} I_{n} \varphi_{q n}\left(\underline{r}_{T}\right)=0 \\
&\left|\sum_{n=1}^{N} I_{n} \Phi_{n}\left(\underline{r}^{\prime}\right)\right|^{2} \leq \mathcal{U} \mathscr{B}(\underline{r}) \\
&
\end{aligned}
$$

where $\mathbf{p}$ is a generic polarization. The solution of the CP problem (7a), (7b), (7c), and (7d) (i.e., the optimal excitations for the polarization at hand) can be easily achieved by offthe-shelf numerical libraries (such as the FMINCON by MATLAB).

Let us define $F(\mathbf{p})$ as the maximum value which is achieved by the cost function (7a) (i.e., the value which is achieved at the end of the CP procedure or when the array is fed by the optimal excitations $\left.\mathbf{I}=\left[I_{1}, \ldots, I_{n}\right]\right)$.

Then, the second proposed strategy simply amounts to optimize (by means of global or even local optimization procedures) the function $F(\mathbf{p})$. Anyway, even if $F(\mathbf{p})$ is optimized by means of global search algorithm, such a strategy allows the reduction of the number of unknowns dealt with in the global optimization process with respect to the simpler solution of performing the global optimization simultaneously on all variables. This is due to the fact 


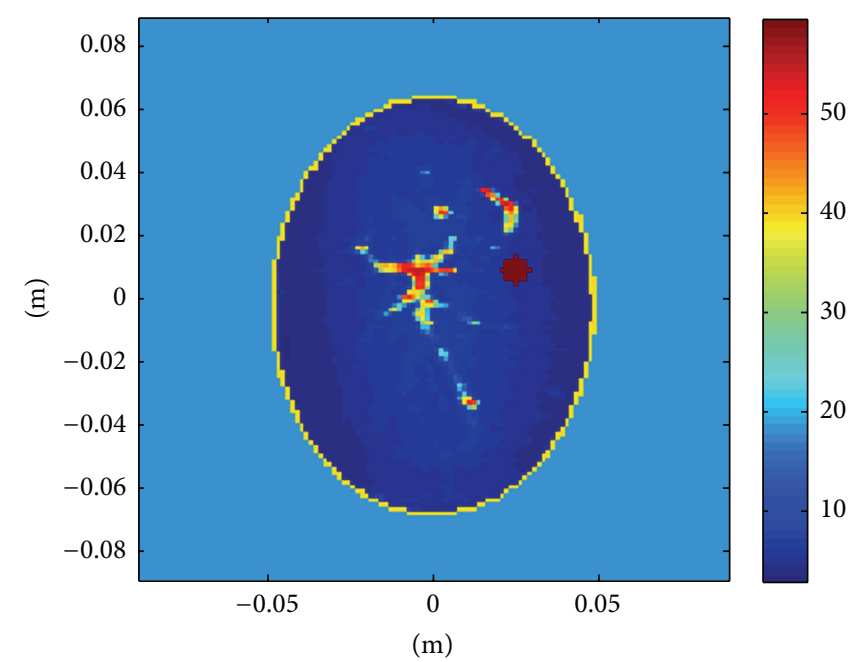

(a)

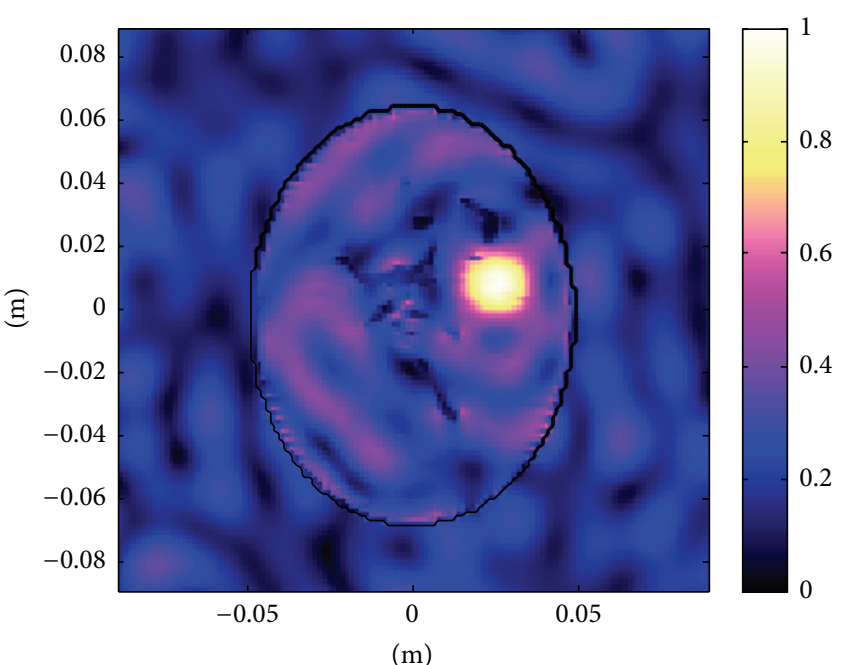

(b)

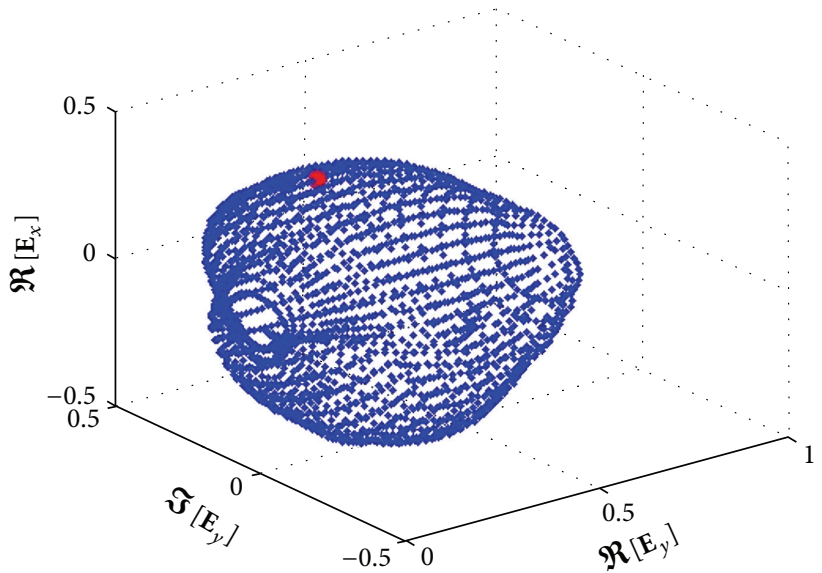

(c)

FIGURE 1: Focusing in a fatty breast phantom (slice 155, ID: 071904) [20, 21]: (a) permittivity profile, (b) intensity of the field |E( $\underline{r}) \mid$ focused into the tumor, and (c) polar plot of the optimal field intensity (i.e., $F(\mathbf{p})$ ), as a function of all possible polarizations $\mathbf{p}$ of the field into the target point performed by the corresponding optimal excitations set $\mathbf{I}=\left[I_{1}, I_{2}, \ldots I_{n}\right]$. The red dot represents the polarization $\left(\mathbf{p}=\left[0.4148,0.9099 e^{-j 0.53 \pi}\right]\right)$ which provides the highest field intensity into the target point $\left|\mathbf{E}\left(\underline{r}_{T}\right)\right|$ for a fixed $\mathscr{U} \mathscr{B}(\underline{r})$ value.

that this approach allows exploiting the convexity of the problem with respect to the excitations while tackling the noconvexity of the optimization in the space of polarization by means of global search algorithms. As a consequence, global optimization tools will have to deal with a reduced number of unknowns, thus saving computational times and avoiding being trapped in local optimal solutions.

A similar strategy is obviously possible for generic 3D vector problems, wherein the optimal plane of polarization has however to be found.

\section{Numerical Results}

The first proposed technique to focus vector fields has been assessed in biological scenarios representing two kinds of anatomically realistic breasts $[20,21]$ affected by an early stage tumor of $4 \mathrm{~mm}$ radius; see Figures 1(a) and 2(a).
The breast is immersed in a matching and/or cooling liquid aimed at reducing reflection at the breast surface and the temperature of the skin or of the surrounding healthy tissues, as the field focusing procedure is meant to be part of hyperthermic treatment [1]. Such a medium is chosen according to the criteria discussed in [22]. By trading off the different requirements, the relative permittivity and the conductivity of the background medium have been set to 18 and $0.1 \mathrm{~S} / \mathrm{m}$, respectively [22]. A matching liquid having these electromagnetic properties can be obtained by mixing a proper concentration of water and Triton X-100 [23].

The applicator is supposed to be a circular array of magnetic currents surrounding the breast, whose radius is $14 \mathrm{~cm}$. The number of the array's elements is set to $N=22$, according to the well-known results on spatial bandwidth of fields [24]. 


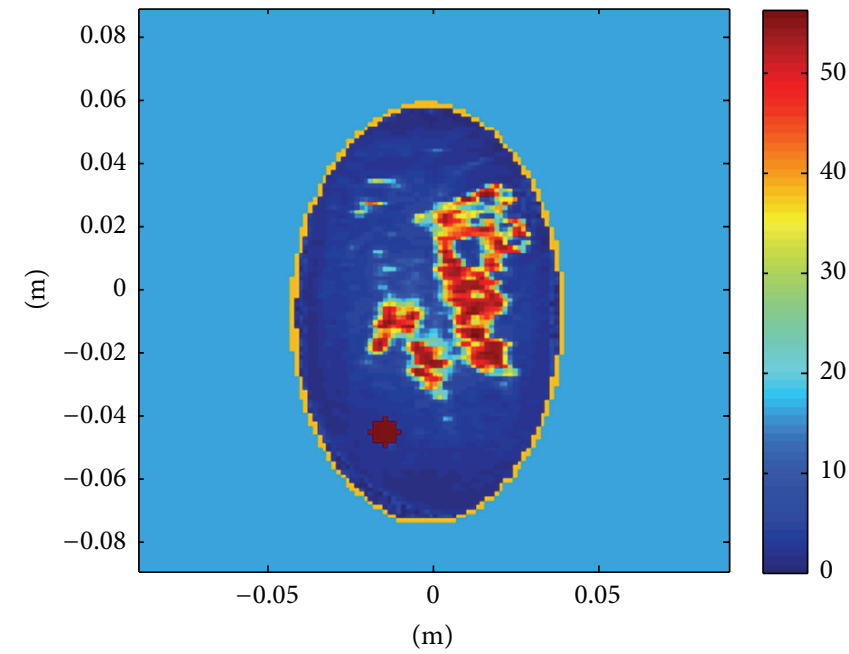

(a)

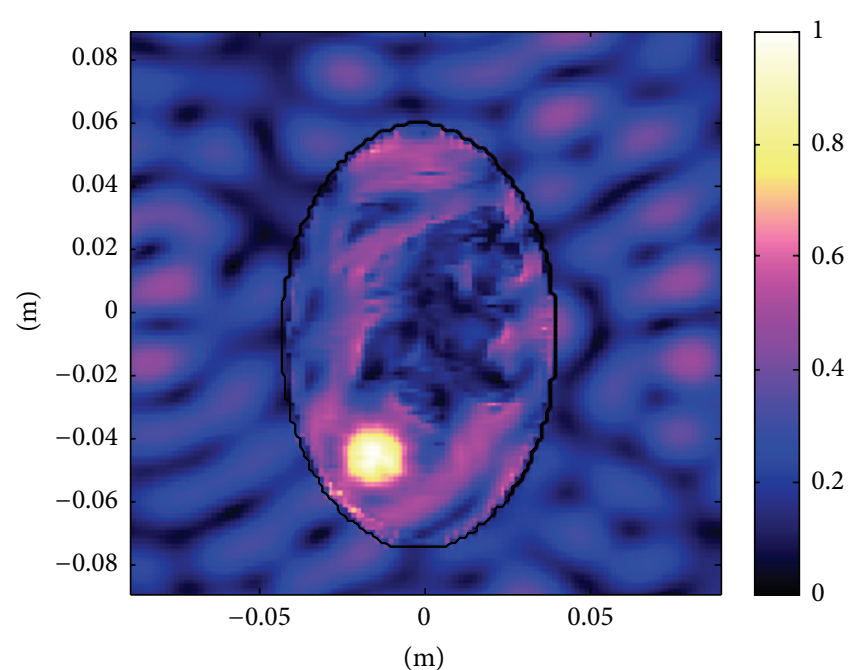

(b)

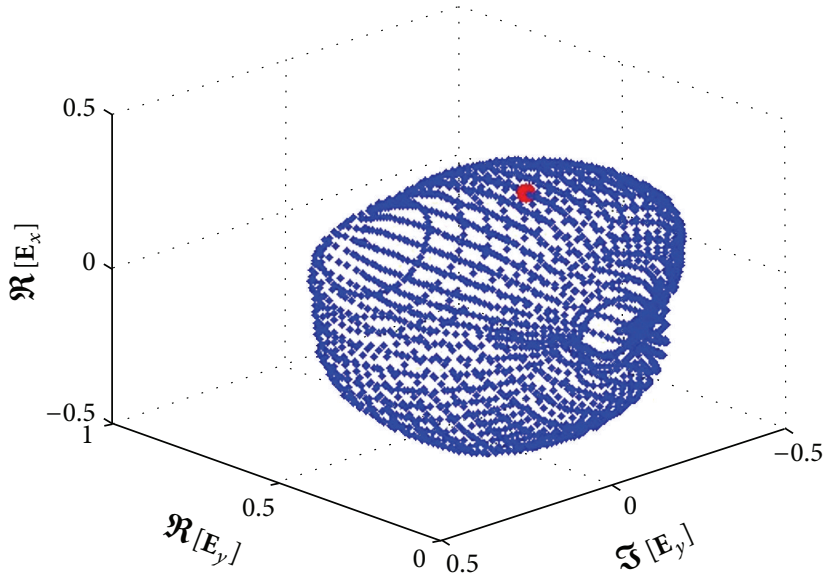

(c)

Figure 2: Focusing in a fatty breast phantom (slice 155, ID: 012304) [20, 21]: (a) permittivity profile, (b) intensity of the field |E( $r) \mid$ focused into the tumor, and (c) polar plot of the optimal field intensity (i.e., $F(\mathbf{p})$ ), as a function of all possible polarizations $\mathbf{p}$ of the field into the target point performed by the corresponding optimal excitations set $\mathbf{I}=\left[I_{1}, I_{2}, \ldots, I_{n}\right]$. The red dot represents the polarization $(\mathbf{p}=$ $\left.\left[0.7886,0.6149 e^{-j 0.49 \pi}\right]\right)$ which provides the highest field intensity into the target point $\left|\mathbf{E}\left(\underline{r}_{T}\right)\right|$ for a fixed $\mathscr{U} \mathscr{B}(\underline{r})$ value.

Figures 1 and 2 report the outcomes of the proposed strategy. In particular, Figures 1(a) and 2(a) report the permittivity profile of the considered realistic breasts and Figures 1(b) and 2(b) report the best result one is able to achieve in terms of field intensity. As it can be seen, the field shaped by the proposed procedure is well focused into the tumor location, and the maximum amplitude of the side lobes is around $50 \%$ and $58 \%$ of the field intensity into the tumor location, in case of fatty and very dense breast, respectively. The second slightly poorer result can be due to the higher anatomical complexity and content of lossy tissues in the considered very dense breast. Notably, these are the globally best field distributions which can be achieved for an a priori fixed $\mathscr{U} \mathscr{B}(\underline{r})$ function, which can be eventually customized in order to perform a patient specific treatment. For the sake of simplicity, in both the considered cases $\mathcal{U} \mathscr{B}(\underline{r})$ has been chosen as a constant function.
Moreover, in order to better understand the rationale of the proposed strategy, we also report the partial outcomes of the optimization procedure in the $3 \mathrm{D}$ space defined by $\mathfrak{R}\left\{\mathbf{E}_{x}\left(\underline{r}_{T}\right)\right\} \mathfrak{R}\left\{\mathbf{E}_{y}\left(\underline{r}_{T}\right)\right\}, \mathfrak{\Im}\left\{\mathbf{E}_{y}\left(\underline{r}_{T}\right)\right\}$ (see Figures 1(c) and 2(c)). In such a space, points of the unitary sphere define all possible polarizations, which also means that each direction departing from the origin defines one of the possible polarizations (since polarization only depends on the relative phases, $\mathbf{E}_{x}\left(\underline{r}_{T}\right)$ can be assumed to be real without any loss of generality). Then, function $F(\mathbf{p})$ as defined in Section 3 can be conveniently represented as a polar plot in such a space, and the optimal polarization will be simply identified by the (red) point which is farthest from the origin (and from its direction).

As it can be seen from Figures 1(c) and 2(c) (showing the polar plots of $F(\mathbf{p})$ ), in both cases the globally best solution corresponds to fields which are elliptically polarized in the tumor point in such a way that cannot be foreseen. 
Also note that these polar diagrams exhibit a smooth behavior as a function of the different polarizations, so that we expect that the second proposed strategy will be able to correctly identify the best possible solutions even in case a local search algorithm is used in the space of polarizations.

\section{Conclusions}

In this paper the problem of focusing a vector field into a nonhomogeneous medium has been tackled by giving a novel insight into Optimal Constrained Power Focusing techniques $[8,9,25]$. In particular, it has been showed that when dialing with vector fields the focusing problem can be recast as an optimization with respect to two sets of unknowns, that is, the polarization of the field into the target point and the arrays' excitations (when considering a fixed array geometry). Moreover, it has been demonstrated that the problem at hand is no-convex/convex with respect to the former/latter set of unknowns. This circumstance leads to the development of two solution strategies. One is based on the solution of all convex programming problems, with respect to the array's excitations, associated with each polarization, in an enumerative fashion. The other one relies on performing the global optimization in the polarization's space.

Both approaches are more computational efficient than applying global optimization algorithms to solve the overall focusing problem, as they exploit the partial convexity of the problem. Moreover the enumerative strategy can take advantage of parallel programming toolboxes to speed up the computation. On the other hand the alternative proposed approach implies the use of global search algorithms just in the space of polarizations unless the cost functional has a smooth behavior, when local search algorithm may be used.

Even though 2D scenarios are considered, the reported results are relevant because they suggest possible ways of improving the OCPF efficiency since the computational burden increases considerably when dealing with 3D scenarios. Finally it is worth noting that the presented theoretical reasoning can be easily extended to $3 \mathrm{D}$ vector focusing that is the ultimate goal of ongoing research activities.

\section{Conflict of Interests}

The author declares that there is no conflict of interests regarding the publication of this paper.

\section{References}

[1] A. J. Fenn, Adaptive Phased Array Thermotherapy for Cancer, Artech House, London, UK, 2008.

[2] J.-L. Thomas, F. Wu, and M. Fink, "Time reversal focusing applied to lithotripsy," Ultrasonic Imaging, vol. 18, no. 2, pp. 106121, 1996.

[3] T. W. Wong, "Use of microwave in processing of drug delivery systems," Current Drug Delivery, vol. 5, no. 2, pp. 77-84, 2008.

[4] M. E. Yavuz and F. L. Teixeira, "Ultrawideband microwave sensing and imaging using time-reversal techniques: a review," Remote Sensing, vol. 1, no. 3, pp. 466-495, 2009.
[5] M. Fink, "Time reversal of ultrasonic fields. I. Basic principles," IEEE Transactions on Ultrasonics, Ferroelectrics, and Frequency Control, vol. 39, no. 5, pp. 555-566, 1992.

[6] M. Tanter, J. L. Thomas, and M. Fink, "Optimal focusing by spatio-temporal inverse filter. I. Basic principles," The Journal of the Acoustical Society of America, vol. 108, pp. 223-234, 2000.

[7] M. Converse, E. J. Bond, B. D. VanVeen, and S. C. Hagness, "A computational study of ultra-wideband versus narrowband microwave hyperthermia for breast cancer treatment," IEEE Transactions on Microwave Theory and Techniques, vol. 54, no. 5, pp. 2169-2180, 2006.

[8] D. A. M. Iero, T. Isernia, A. F. Morabito, I. Catapano, and L. Crocco, "Optimal constrained field focusing for hyperthermia cancer therapy: a feasibility assessment on realistic phantoms," Progress in Electromagnetics Research, vol. 102, pp. 125-141, 2010.

[9] D. A. M. Iero, L. Crocco, and T. Isernia, "Thermal and microwave constrained focusing for patient-specific breast cancer hyperthermia: a robustness assessment," IEEE Transactions on Antennas and Propagation, vol. 62, no. 2, pp. 814-821, 2014.

[10] D. A. M. Iero, T. Isernia, and L. Crocco, "Focusing timeharmonic scalar fields in complex scenarios: a comparison," IEEE Antennas and Wireless Propagation Letters, vol. 12, pp. 1029-1032, 2013.

[11] D. A. M. Iero, T. Isernia, and L. Crocco, "Focusing time harmonic scalar fields in non-homogenous lossy media: inverse filter vs. constrained power focusing optimization," Applied Physics Letters, vol. 103, no. 9, Article ID 093702, 2013.

[12] E. Zastrow, S. C. Hagness, and B. D. Van Veen, "3D computational study of non-invasive patient-specific microwave hyperthermia treatment of breast cancer," Physics in Medicine and Biology, vol. 55, no. 13, pp. 3611-3629, 2010.

[13] D. G. Luenberger and Y. Ye, Linear and Nonlinear Programming, International Series in Operations Research \& Management Science, Springer, 3rd edition, 2008.

[14] J. Robinson and Y. Rahmat-Samii, "Particle swarm optimization in electromagnetics," IEEE Transactions on Antennas and Propagation, vol. 52, no. 2, pp. 397-407, 2004.

[15] T. Isernia and G. Panariello, "Optimal focusing of scalar fields with arbitrary upper bounds," in Atti XI Riunione Nazionale di Elettromagnetismo (XI RiNEm '96), Florence, Italy, October 1996 (Italian).

[16] T. Isernia and G. Panariello, "Optimal focusing of scalar fields subject to arbitrary upper bounds," Electronics Letters, vol. 34, no. 2, pp. 162-164, 1998.

[17] G. Franceschetti, Electromagnetics: Theory, Techniques, and Engineering Paradigms, Springer, New York, NY, USA, 1997.

[18] D. H. Wolpert and W. G. Macready, "No free lunch theorems for optimization," IEEE Transactions on Evolutionary Computation, vol. 1, no. 1, pp. 67-82, 1997.

[19] M. D'Urso and T. Isernia, “Solving some array synthesis problems by means of an effective hybrid approach," IEEE Transactions on Antennas and Propagation, vol. 55, no. 3, pp. 750-759, 2007.

[20] E. Zastrow, S. K. Davis, M. Lazebnik, F. Kelcz, B. D. Van Veen, and S. C. Hagness, "Development of anatomically realistic numerical breast phantoms with accurate dielectric properties for modeling microwave interactions with the human breast," IEEE Transactions on Biomedical Engineering, vol. 55, no. 12, pp. 2792-2800, 2008.

[21] E. Zastrow, S. K. Davis, M. Lazebnik, F. Kelcz, B. D. van Veem, and S. C. Hagness, Database of 3D grid-based numerical 
breast phantom for use in computational electromagnetics simulations, http://uwcem.ece.wisc.edu/home.htm.

[22] I. Catapano, L. Di Donato, L. Crocco et al., "On quantitative microwave tomography of female breast," Progress in Electromagnetics Research, vol. 97, pp. 75-93, 2009.

[23] S. Romeo, L. Di Donato, O. M. Bucci et al., "Dielectric characterization study of liquid-based materials for mimicking breast tissues," Microwave and Optical Technology Letters, vol. 53, no. 6, pp. 1276-1280, 2011.

[24] O. M. Bucci and T. Isernia, "Electromagnetic inverse scattering: retrievable information and measurement strategies," Radio Science, vol. 32, no. 6, pp. 2123-2137, 1997.

[25] D. A. M. Iero, L. Crocco, and T. Isernia, "Constrained power focusing of vector fields: an innovative globally optimal strategy," Journal of Electromagnetic Waves and Applications, vol. 29, no. 13, pp. 1708-1719, 2015. 

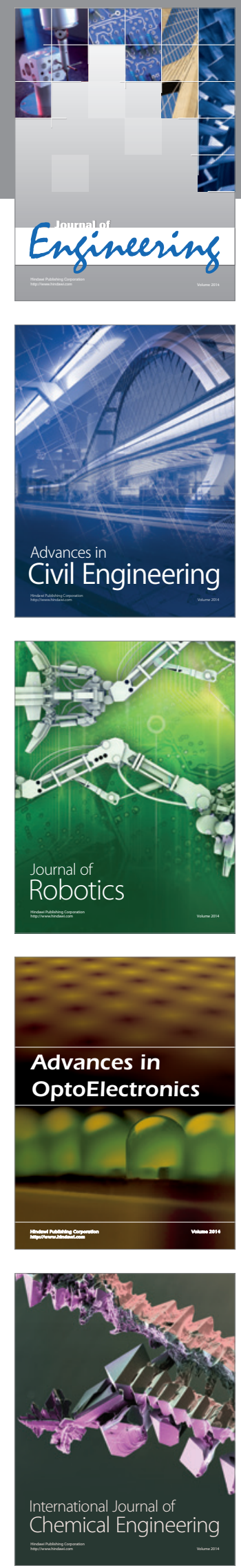

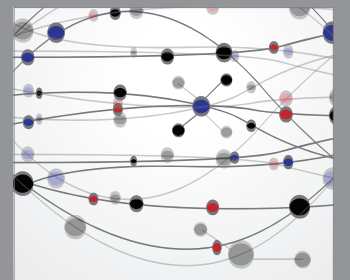

The Scientific World Journal
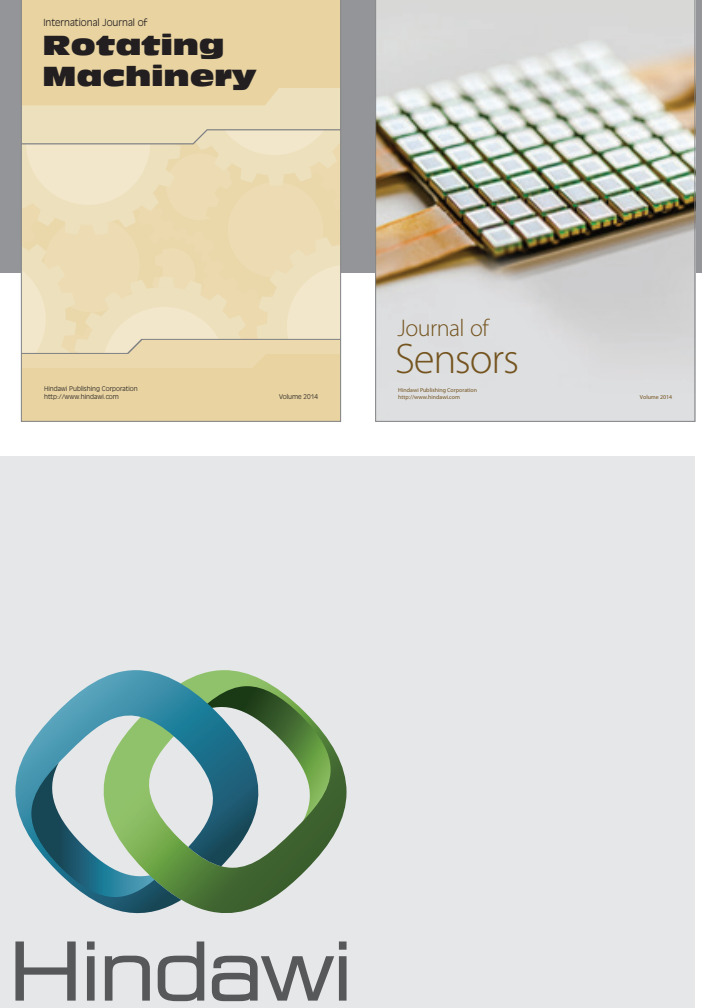

Submit your manuscripts at http://www.hindawi.com
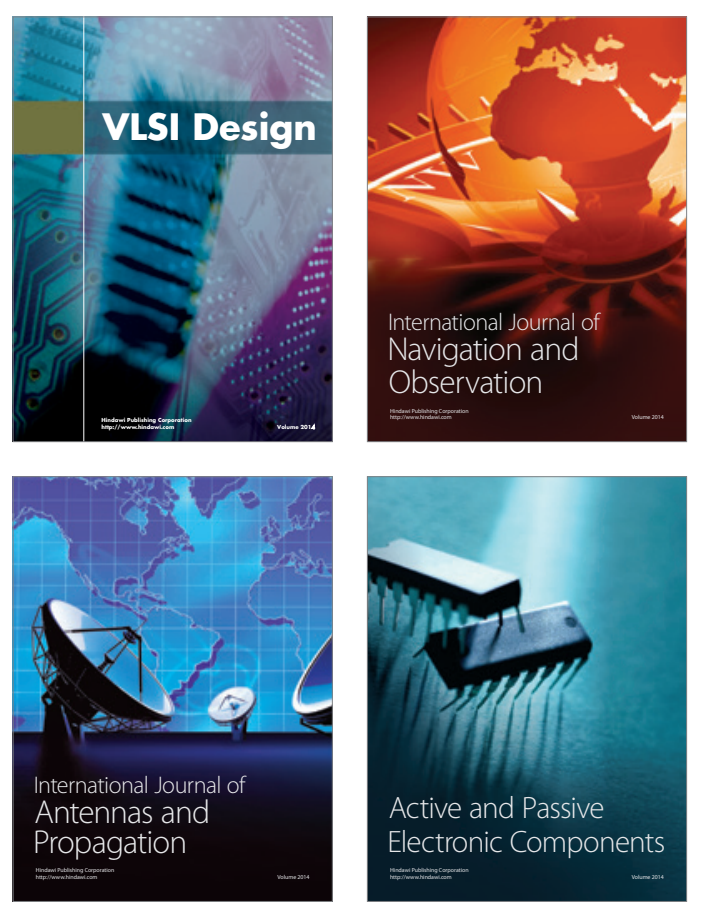
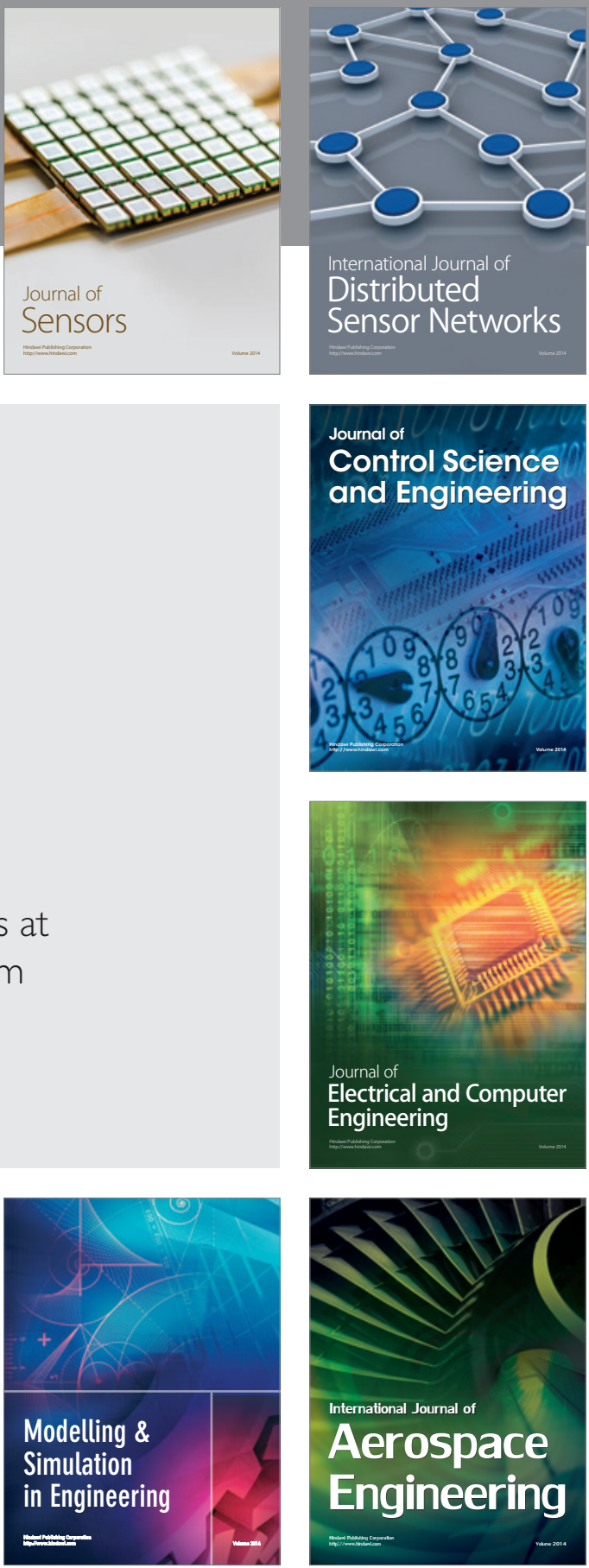

Journal of

Control Science

and Engineering
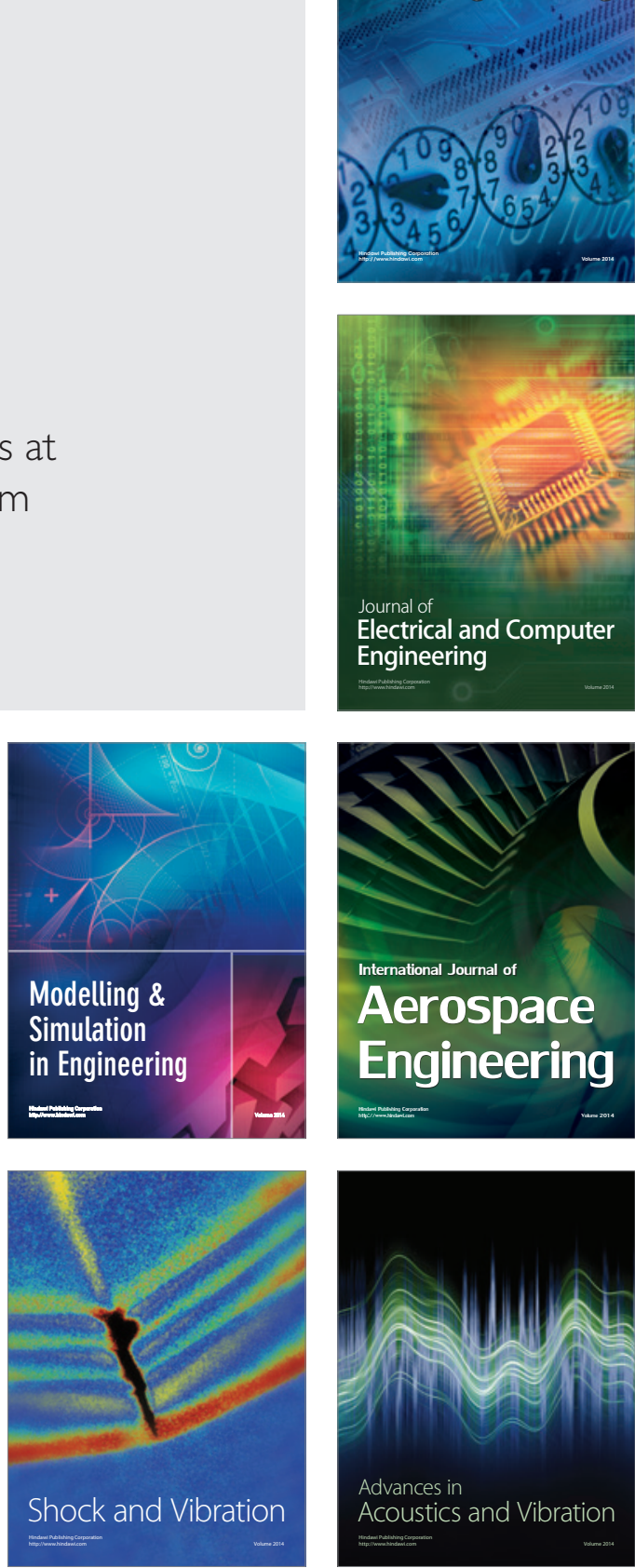